\title{
TORSION FREE GROUPS
}

\author{
PAUL HILL ${ }^{1}$ AND CHARLES MEGIBBEN
}

\begin{abstract}
In this paper we introduce the class of torsion free $k$-groups and the notion of a knice subgroup. Torsion free $k$-groups form a class of groups more extensive than the separable groups of Baer, but they enjoy many of the same closure properties. We establish a role for knice subgroups of torsion free groups analogous to that played by nice subgroups in the study of torsion groups. For example, among the torsion free groups, the balanced projectives are characterized by the fact that they satisfy the third axiom of countability with respect to knice subgroups. Separable groups are characterized as those torsion free $k$-groups with the property that all finite rank, pure knice subgroups are direct summands. The introduction of these new classes of groups and subgroups is based on a preliminary study of the interplay between primitive elements and *-valuated coproducts. As a by-product of our investigation, new proofs are obtained for many classical results on separable groups. Our techniques lead naturally to the discovery that a balanced subgroup of a completely decomposable group is itself completely decomposable provided the corresponding quotient is a separable group of cardinality not exceeding $\aleph_{1}$; that is, separable groups of cardinality $\aleph_{1}$ have balanced projective dimension $\leq 1$.
\end{abstract}

1. Introduction. In this paper, we examine the fundamental concepts underlying the theory of torsion free abelian groups. This is done in the spirit of the seminal work of Baer [1] and involves a reappraisal of some of the most basic notions in the light of new ideas introduced in our recent third axiom of countability characterization of $p$-local Warfield groups in [8]. Indeed the present paper should be viewed in the context of our ongoing study of isotype subgroups of simply presented groups initiated in [7]. In particular, we generalize Baer's notion of a primitive element which, when coupled with our concept of a *-valuated coproduct, leads not only to new results but also to new proofs of a number of classical theorems.

Throughout this paper, $G$ denotes an additively written torsion free abelian group. By a height sequence we understand a sequence $s=\left(s_{p}\right)_{p \in \mathbf{P}}$, indexed by the set $\mathbf{P}$ of primes, where each $s_{p}$ is either a nonnegative integer or the symbol $\infty$. Height sequences are, of course, ordered pointwise and in fact form a complete distributive lattice with the meet operation defined by $s \wedge t=\left(s_{p} \wedge t_{p}\right)_{p \in \mathbf{P}}$ where $s_{p} \wedge t_{p}=\min \left\{s_{p}, t_{p}\right\}$. With each $x \in G$, we associate its height sequence $|x|$, where $|x|_{p}$ is the height in $G$ of $x$ at the prime $p$, that is, $|x|_{p}=n$ if $x \in p^{n} G \backslash p^{n+1} G$ and $|x|_{p}=\infty$ if $x \in p^{n} G$ for all $n<\omega$. Each height sequence $s$ determines a fully

Received by the editors June 18,1985 . Presented to the Society at the 92nd annual meeting, New Orleans, Louisiana, January 7-11, 1986.

1980 Mathematics Subject Classification. Primary 20K15, $20 \mathrm{~K} 20$.

Key words and phrases. Torsion free group, primitive element, *-valuated coproduct, free *-valuated subgroup, $k$-group, separable group, knice subgroup, completely decomposable, third axiom of countability, balanced projective dimension.

${ }^{1}$ Research supported, in part, by N.S.F. Grant \#MCS-8102470. 
invariant subgroup $G(s)=\{x \in G:|x| \geq s\}$. Notice that $s \leq t$ implies $G(s) \supseteq G(t)$. Recall that height sequences $s$ and $t$ are said to be equivalent provided (i) $s_{p}=t_{p}$ for all but finitely many $p$ and (ii) $s_{p}=\infty$ if and only if $t_{p}=\infty$. Agreeing that $\infty-\infty=0$, we observe that $s$ and $t$ are equivalent if and only if $\sum_{p \in \mathbf{P}}\left|s_{p}-t_{p}\right|$ is finite. It is, of course, clear that $|x|$ and $|y|$ are equivalent if there exist nonzero integers $m$ and $n$ such that $m x=n y$. An equivalence class of height sequences is called a type, and the lattice relations among height sequences induce in the obvious manner corresponding relations on the set of types. In a rank one group (i.e., a subgroup of the additive group of rationals), all nonzero elements are of the same type. We also find it convenient to define a multiplication of height sequences by positive integers as follows: $n s$ is the height sequence $\left(t_{p}\right)_{p \in \mathbf{P}}$ given by $t_{p}=s_{p}+n_{p}$, where $p^{n_{p}}$ is the highest power of $p$ dividing $n$. Thus $s$ and $t$ are equivalent if and only if there exist positive integers $m$ and $n$ such that $m s=n t$. Notice that $G(n s)=$ $n G(s)$. In addition to the subgroup $G(s)$, we shall also require the fully invariant subgroup $G\left(s^{*}\right)$ generated by those $x \in G(s)$ such that $|x|$ is not equivalent to $s$; i.e., each element of $G\left(s^{*}\right)$ is a sum of elements $x \in G(s)$ with $\sum_{p \in \mathbf{P}}\left(|x|_{p}-s_{p}\right)=\infty$. If $t=n s$, then $G\left(t^{*}\right)=n G\left(s^{*}\right)$. Occasionally we shall need to consider the fully invariant subgroup $G(\sigma)=\sum_{s \in \sigma} G(s)=\bigcup_{s \in \sigma} G(s)$ determined by the type $\sigma$, as well as the similarly defined $G\left(\sigma^{*}\right)=\sum_{s \in \sigma} G\left(s^{*}\right)=\bigcup_{s \in \sigma} G\left(s^{*}\right)$.

Finally, recall that a torsion free group $G$ is said to be completely decomposable if it decomposes into a direct sum of rank one subgroups, and that $G$ is separable (in the sense of Baer) if each finite subset of $G$ can be imbedded in a finite rank completely decomposable direct summand.

2. Primitive elements and *-valuated coproducts. Our notion of a primitive element $x$ in $G$ is motivated by the requirement that when $G$ is completely decomposable, the pure subgroup $\langle x\rangle_{*}$ generated by $x$ be a summand. Baer's requirement that a primitive element $x$ of type $\sigma$ satisfy $|x| \geq|x+g|$ for all $g \in G\left(\sigma^{*}\right)$ is, however, too stringent and is indeed not appropriate for groups which fail to be separable. To provide insight into our more general definition of primitivity, we consider a simple example. Suppose $G=A \oplus B$ is a rank two group containing elements $a \in A$ and $b \in B$ such that $|a|=(1,1,1, \ldots)$ and $|b|=(0, \infty, 1, \ldots)$. Let $x=a+b$ and $y=a^{\prime}+b$, where $2 a^{\prime}=a$. Then both $x$ and $y$ have $s=(0,1,1, \ldots)$ as their height sequence. But $\langle y\rangle_{*}$ is a direct summand of $G$ with $B$ serving as a complement (see 2.6 below), while the pure subgroup $\langle x\rangle_{*}$ fails to be a summand of $G$. The source of this difference between $x$ and $y$ resides in the fact that $b$ is an element of $G\left(s^{*}\right)$ such that $x-b$ has greater height than $x$ at the prime $p=2$. Agreeing to write $G\left(s^{*}, p\right)$ for $G\left(s^{*}\right)+G(p s)$ and being guided by the foregoing example, we now formulate our version of primitivity.

DEFINITION 2.1. Let $x$ be an element of the torsion free group $G$. If $x \notin G\left(s^{*}, p\right)$ for each prime $p$ and each height sequence $s$ equivalent to $|x|$ for which $s_{p}=|x|_{p}$ and $|x|_{p} \neq \infty$, then we say that $x$ is primitive in $G$.

If $\langle x\rangle_{*}$ is a direct summand of $G$, then it is trivial to verify that $x$ is primitive. Moreover, it is not difficult, using well-known facts about finite rank completely decomposable groups, to give an ad hoc proof that $\langle x\rangle_{*}$ is a summand provided $x$ is primitive in the completely decomposable group $G$. But this latter observation arises quite naturally in the general developments to follow (see 2.9 below). Nutire that 0 is primitive, as is any $x$ with $|x|_{p}=\infty$ for all primes $p$. A simple and 
frequently useful observation about primitive elements is the following: If $x$ is primitive in $G$ and if $x \in G\left(s^{*}, p\right)$, then either $\sum_{p \in \mathbf{P}}\left(|x|_{p}-s_{p}\right)=\infty$ or $x \in G(p s)$.

LEMMA 2.2. Let $x \in G$ and suppose $n$ is a nonzero integer. Then $x$ is primitive in $G$ if and only if $n x$ is primitive in $G$.

PROOF. That $n x$ being primitive implies the same for $x$ is a consequence of the fact that $G\left(t^{*}, p\right)=n G\left(s^{*}, p\right)$ if $t=n s$. Conversely, assume that $x$ is primitive and observe that it suffices to consider the case where $n$ is a prime. Assume by way of contradiction that $n x \in G\left(s^{*}, p\right)$, where $s$ is equivalent to $|n x|$ and $s_{p}=|n x|_{p} \neq \infty$. If $n=p$, then $|n x|_{p}=|x|_{p}+1$ and there is a height sequence $t$ such that $p t=s$ and $t_{p}=|x|_{p}$. In this case, $n x=p x \in G\left(s^{*}, p\right)=p G\left(t^{*}, p\right)$ and $x \in G\left(t^{*}, p\right)$, contradicting the primitivity of $x$. Suppose, however, that $n \neq p$ and choose integers $l$ and $m$ such that $1=l p+m n$. Clearly there is a height sequence $t$ equivalent to $s$ such that $t \leq|x|, t \leq s$ and $t_{p}=s_{p}=|n x|_{p}=|x|_{p}$. Then $x=l p x+m n x$ is in $G(p t)+G\left(s^{*}, p\right) \subseteq G\left(t^{*}, p\right)$, once again contradicting the fact that $x$ is primitive.

LEMMA 2.3. If $x$ is primitive in $G$ with $s=|x|$, then each element of the coset $x+G\left(s^{*}\right)$ is primitive with $s$ as its height sequence.

PROOF. Let $y=x+z$, where $z \in G\left(s^{*}\right)$. Assume by way of contradiction that $y \in G\left(t^{*}, p\right)$, where $t$ is equivalent to $|y|$ and $t_{p}=|y|_{p} \neq \infty$. Notice that $|y| \geq$ $|x| \wedge|z| \geq s$ and hence $t_{p} \geq s_{p}$. Then $x=y-z \in G\left(t^{*}, p\right)+G\left(s^{*}\right) \subseteq G\left((s \wedge t)^{*}, p\right)$. But this contradicts the fact that $x$ is primitive since $s \wedge t$ is equivalent to $|x|$ and $s_{p} \wedge t_{p}=s_{p}=|x|_{p}$. Finally, observe that $|y|_{p}>|x|_{p} \neq \infty$ for some prime $p$ would also contradict the primitivity of $x$.

Having settled on our definition of primitive element, the next problem we wish to consider is the formulation of a suitably general condition relating two independent primitive elements so that the pure subgroup generated by them will be a summand when the containing group is completely decomposable. As Baer [1] observed, using his notion of primitivity, there is no real difficulty if the elements are of different types. The case when the primitive elements are of the same type, however, seems not to have been dealt with successfully in the literature.

Consider the direct sum $A=\left\langle x_{1}\right\rangle \oplus\left\langle x_{2}\right\rangle$, where $x_{1}$ and $x_{2}$ are independent primitive elements in $G$. If there is any hope for $B=\left\langle x_{1}\right\rangle_{*} \oplus\left\langle x_{2}\right\rangle_{*}$ to be a direct summand of $G$, then we must have $\left|n_{1} x_{1}+n_{2} x_{2}\right|=\left|n_{1} x_{1}\right| \wedge\left|n_{2} x_{2}\right|$ for all integers $n_{1}$ and $n_{2}$. This observation leads naturally to the following definition. A direct sum $A_{1} \oplus A_{2}$ of independent subgroups of $G$ is said to be a valuated coproduct in $G$ if $\left|a_{1}+a_{2}\right|=\left|a_{1}\right| \wedge\left|a_{2}\right|$ for all $a_{1} \in A$ and $a_{2} \in A_{2}$. An equivalent formulation is that $a_{1}+a_{2} \in G(s)$ implies $a_{1}, a_{2} \in G(s)$ for all height sequences $s$. This definition generalizes in the obvious manner to arbitrary direct sums $\bigoplus_{i \in I} A_{i}$ of independent subgroups of $G$. If $B_{i} / A_{i}$ is torsion for all $i$, then $\bigoplus_{i \in I} B_{i}$ is a valuated coproduct in $G$ if and only if $\bigoplus_{i \in I} A_{i}$ is. Indeed this follows from the fact that $G$ is torsion free and $G(n s)=n G(s)$ for all $n$ and $s$. Another easy but important observation is the fact that the valuated coproduct $\bigoplus_{i \in I} A_{i}$ is pure in $G$ if each $A_{i}$ is a pure subgroup of $G$. The requirement that $A=\left\langle x_{1}\right\rangle \oplus\left\langle x_{2}\right\rangle$ be a valuated coproduct in $G$ does not suffice, however, to make $B=\left\langle x_{1}\right\rangle_{*} \oplus\left\langle x_{2}\right\rangle_{*}$ a direct summand of the completely decomposable group $G$. To understand why this is so, we consider another example. 
Let $G=A_{1} \oplus A_{2} \oplus A_{3}$ be a rank three completely decomposable group containing elements $x_{1} \in A_{1}, x_{2} \in A_{2}$ and $x_{3} \in A_{3}$ such that $\left|x_{1}\right|=(0,0,0, \ldots),\left|x_{2}\right|=$ $(\infty, 0,0, \ldots)$ and $\left|x_{3}\right|=(0, \infty, 0, \ldots)$. Then $y=x_{1}+x_{2}+x_{3}$ is a primitive element by Lemma 2.3 with $|y|=\left|x_{1}\right|$, and $A=\left\langle x_{1}\right\rangle \oplus\langle y\rangle$ is readily seen to be a valuated coproduct in $G$. Nonetheless, the pure subgroup $B=\left\langle x_{1}\right\rangle_{*} \oplus\langle y\rangle_{*}$ fails to be a direct summand of $G$. Indeed, well-known and easily proved facts about summands of completely decomposable groups forbid $G$ having a direct summand isomorphic to $B$. A more intrinsic insight into the failure of $B$ to be a summand of $G$ is gained from the observation that $y-x_{1}=x_{2}+x_{3} \in G\left(s^{*}\right)$, where $s=|y|=\left|x_{1}\right|$ and neither $y$ nor $x_{1}$ is in $G\left(s^{*}\right)$. Employing the subgroups $G\left(s^{*}, p\right)$ again, we show that the defect observed in the preceding example can be overcome by the introduction of a notion more stringent than that of a valuated coproduct.

DEFINITION 2.4. Let $A=\bigoplus_{i \in I} A_{i}$ be a valuated coproduct in $G$ and represent each $a \in A$ as a sum $a=\sum_{i \in I} a_{i}$, where $a_{i} \in A_{i}$ for all $i$. If for each prime $p$ and each height sequence $s$ it is the case that $a \in G\left(s^{*}\right)$ implies $a_{i} \in G\left(s^{*}\right)$ for all $i$ and also $a \in G\left(s^{*}, p\right)$ implies $a_{i} \in G\left(s^{*}, p\right)$ for all $i$, then we say that $A=\bigoplus_{i \in I} A_{i}$ is a *-valuated coproduct.

Observe that in the special case of a valuated coproduct $A=A_{1} \oplus A_{2}$, where $A_{2}=\langle x\rangle$ with $x$ primitive in $G$, one needs only verify the latter of the two conditions to establish that $A=A_{1} \oplus A_{2}$ is a $*$-valuated coproduct. Once again, if $B_{i} / A_{i}$ is torsion for all $i$, the direct sum $\bigoplus_{i \in I} B_{i}$ is a *-valuated coproduct in $G$ if and only if $\bigoplus_{i \in I} A_{i}$ is a $*$-valuated coproduct in $G$. The notion of a $*$-valuated coproduct provides us with precisely the right tool to resolve the question of when the pure subgroup generated by two independent primitive elements is a summand of the completely decomposable group containing them. To see that this is so, however, requires a series of lemmas.

LEMMA 2.5. If $N \oplus\langle x\rangle$ is a *-valuated coproduct in $G$ with $x$ primitive and if $y=x+z$, where $z \in N$ and $|y|=|x|$, then $y$ is primitive and $N \oplus\langle y\rangle$ is a $*$-valuated coproduct in $G$.

PROOF. First observe that $N \oplus\langle x\rangle$ being a $*$-valuated coproduct forces $y=x+z$ to be primitive since $y \in G\left(s^{*}, p\right)$ implies $x \in G\left(s^{*}, p\right)$, where $|y|=|x|$. To show that $N \oplus\langle y\rangle$ is at least a valuated coproduct, it is enough to verify that $|w+n y| \leq|n y|$ whenever $w \in N$. But $|w+n y|=|w+n z| \wedge|n x| \leq|n x|=|n y|$. It remains to argue that $w+n y \in G\left(s^{*}, p\right)$ implies $n y \in G\left(s^{*}, p\right)$ whenever $w \in N$. Since $w+n y=(w+n z)+n x$, we have $n x \in G\left(s^{*}, p\right)$. But $n x$ is primitive and hence either $\sum_{p \in \mathbf{P}}\left(|n x|_{p}-s_{p}\right)=\infty$ or $|n x|_{p}>s_{p}$. Because $|y|=|x|$, the first possibility implies $n y \in G\left(s^{*}\right)$ and the second implies $n y \in G(p s)$. In either case, $n y \in G\left(s^{*}, p\right)$.

COROLlaRY 2.6. If $G=\langle x\rangle_{*} \oplus K$, where $|x|=s$, and if $y=x+z$ with $z \in G\left(s^{*}\right)$, then $G=\langle y\rangle_{*} \oplus K$.

Proof. Notice that $N=\langle x\rangle \oplus K$ is a $*$-valuated coproduct with $G / N$ torsion. Since $x=y-z$ is primitive with $z \in G\left(s^{*}\right) \subseteq K,|x|=|y|$ and therefore $N=\langle y\rangle \oplus K$ is a valuated coproduct by 2.5 . Since $\langle y\rangle_{*} \oplus K$ is a pure subgroup of $G$, the desired conclusion follows.

The next technical lemma is crucial to all that follows. 
LEMMA 2.7. Suppose $N=\left\langle x_{1}\right\rangle \oplus\left\langle x_{2}\right\rangle \oplus \cdots \oplus\left\langle x_{n}\right\rangle$ is a *-valuated coproduct in $G$, where $x_{1}, x_{2}, \ldots, x_{n}$ are all primitive elements of the same type. Then every element of $N$ is primitive in $G$. Moreover, if $y_{1}=x_{1}+x_{2}+\cdots+x_{n}$, then there exist elements $y_{2}, \ldots, y_{n}$ in $N$ such that $N=\left\langle y_{1}\right\rangle \oplus\left\langle y_{2}\right\rangle \oplus \cdots \oplus\left\langle y_{n}\right\rangle$ is a $*$-valuated coproduct in $G$.

Proof. A straightforward induction reduces the proof to the case $n=2$. By Lemma 2.2, the first assertion will follow once we show that $y_{1}=x_{1}+x_{2}$ is primitive. Assume by way of contradiction that $y_{1} \in G\left(s^{*}, p\right)$, where $s$ is equivalent to $\left|y_{1}\right|=\left|x_{1}\right| \wedge\left|x_{2}\right|$ and $s_{p}=\left|y_{1}\right|_{p} \neq \infty$. We may suppose without loss of generality that $\left|x_{1}\right|_{p} \leq\left|x_{2}\right|_{p}$. But then the fact that $N=\left\langle x_{1}\right\rangle \oplus\left\langle x_{2}\right\rangle$ is a $*$-valuated coproduct with $\left|x_{1}\right|$ and $\left|x_{2}\right|$ equivalent implies that $x_{1} \in G\left(s^{*}, p\right)$, where $s_{p}=\left|x_{1}\right|_{p}$ and $s$ is equivalent to $\left|x_{1}\right|$. This, however, contradicts the hypothesis that $x_{1}$ is primitive.

Since $\left|x_{1}\right|$ and $\left|x_{2}\right|$ are equivalent, the sets $A_{1}=\left\{p \in \mathbf{P}:\left|x_{1}\right|_{p}<\left|x_{2}\right|_{p} \neq \infty\right\}$ and $A_{2}=\left\{p \in \mathbf{P}:\left|x_{2}\right|_{p}<\left|x_{1}\right|_{p} \neq \infty\right\}$ are finite. Therefore there are relatively prime integers $m$ and $n$ such that $\left|m x_{1}\right|_{p}=\left|x_{2}\right|_{p}$ for all $p \in A_{1}$ and $\left|n x_{2}\right|_{p}=\left|x_{1}\right|_{p}$ for all $p \in A_{2}$. In fact, we may make such a choice with $m$ divisible only by primes in $A_{1}$ and $n$ divisible only by primes in $A_{2}$. Now select integers $k$ and $l$ such that $1=k m+\ln$ and let $y_{2}=-k m x_{1}+\ln x_{2}$. If $p \in A_{1}$, then $\left|y_{1}\right|_{p}=\left|x_{1}\right|_{p}$ and

$$
\left|y_{2}\right|_{p}=\left|k m x_{1}\right|_{p} \wedge\left|\ln x_{2}\right|_{p}=\left|k x_{2}\right|_{p} \wedge\left|l x_{2}\right|_{p}=\left|x_{2}\right|_{p}
$$

since $k$ and $l$ are relatively prime. Similarly, if $p \in A_{2}$, then $\left|y_{1}\right|_{p}=\left|x_{2}\right|_{p}$ and $\left|y_{2}\right|_{p}=\left|x_{1}\right|_{p}$; while if $p \notin A_{1} \cup A_{2}$, the four elements $x_{1}, x_{2}, y_{1}$ and $y_{2}$ all have the same height at $p$. Since the matrix of the transformation between the $y_{i}$ 's and $x_{i}$ 's is unimodular, we have $N=\left\langle y_{1}\right\rangle \oplus\left\langle y_{2}\right\rangle$. We shall first show that this direct sum is at least a valuated coproduct. We need to argue that $\left|n_{1} y_{1}+n_{2} y_{2}\right|_{p}$ equals $\left|n_{1} y_{1}\right|_{p} \wedge\left|n_{2} y_{2}\right|_{p}$ for all integers $n_{1}, n_{2}$ and all primes $p$. Of course, we need only verify this equality under the assumption that $\left|n_{1} y_{1}\right|_{p}=\left|n_{2} y_{2}\right|_{p}$. Moreover, since $G$ is torsion free, common $p$-power factors can be canceled and hence we may assume that at least one of the integers $n_{1}$ or $n_{2}$ is prime to $p$. But since $\left|y_{1}\right|_{p}<\left|y_{2}\right|_{p}$ for $p \in A_{1} \cup A_{2}$ and $\left|y_{1}\right|_{p}=\left|y_{2}\right|_{p}$ for $p \notin A_{1} \cup A_{2}$, we see that $p \mid n_{1}$ if $p \in A_{1} \cup A_{2}$ and that both $n_{1}$ and $n_{2}$ may be taken to be prime to $p$ when $p \notin A_{1} \cup A_{2}$. Now observe that for any $p$ we have $\left|n_{1} y_{1}+n_{2} y_{2}\right|_{p}=\left|m_{1} x_{1}\right|_{p} \wedge\left|m_{2} x_{2}\right|_{p}$, where $m_{1}=n_{1}-k m n_{2}$, $m_{2}=n_{1}+l n n_{2}$ and $m_{2}-m_{1}=n_{2}$. If $p \in A_{1},\left|n_{1} x_{1}\right|_{p}=\left|n_{1} y_{1}\right|_{p}=\left|n_{2} y_{2}\right|_{p}=$ $\left|y_{2}\right|_{p}=\left|x_{2}\right|_{p}$ and thus the same power of $p$ divides $n_{1}$ as divides $m$; that is, at least that power of $p$ divides $m_{1}$ and consequently $p$ does not divide $m_{2}$. Therefore, for $p \in A_{1}$,

$$
\left|m_{2} x_{2}\right|_{p}=\left|n_{2} y_{2}\right|_{p}=\left|n_{1} y_{1}\right|_{p} \leq\left|m_{1} x_{1}\right|_{p}
$$

For $p \in A_{2}$, a similar analysis shows that

$$
\left|m_{1} x_{1}\right|_{p}=\left|n_{2} y_{2}\right|_{p}=\left|n_{1} y_{1}\right|_{p} \leq\left|m_{2} x_{2}\right|_{p} .
$$

On the other hand, if $p \neq A_{1} \cup A_{2}$, the equation $m_{2}-m_{1}=n_{2}$ insures that $p$ divides at most one of the integers $m_{1}$ and $m_{2}$, and consequently in this case we also have $\left|m_{1} x_{1}\right|_{p} \wedge\left|m_{2} x_{2}\right|_{p}=\left|n_{1} y_{1}\right|_{p} \wedge\left|n_{2} y_{2}\right|_{p}$.

Finally, it remains to explain why $N=\left\langle y_{1}\right\rangle \oplus\left\langle y_{2}\right\rangle$ is actually a $*$-valuated coproduct. Suppose that $0 \neq y=n_{1} y_{1}+n_{2} y_{2} \in G\left(s^{*}, p\right)$ and recall that $y$ is necessarily primitive. Then either $y$ has type strictly greater than that determined by $s$ or else $y \in G(p s)$. Since $N=\left\langle y_{1}\right\rangle \oplus\left\langle y_{2}\right\rangle$ is a valuated coproduct, the first 
possibility forces both $n_{1} y_{1}$ and $n_{2} y_{2}$ to be in $G\left(s^{*}\right)$, while the second implies that both are in $G(p s)$.

We now have all the ingredients required to establish the following important exchange property.

THEOREM 2.8. Suppose $N=\left\langle x_{1}\right\rangle \oplus\left\langle x_{2}\right\rangle \oplus \cdots \oplus\left\langle x_{m}\right\rangle$ is a *-valuated coproduct in $G$ where each of the $x_{i}$ 's is primitive. If $y_{1} \neq 0$ is a primitive element contained in $N$, then there exist primitive elements $y_{2}, \ldots, y_{m}$ such that $N^{\prime}=\left\langle y_{1}\right\rangle \oplus\left\langle y_{2}\right\rangle \oplus$ $\cdots \oplus\left\langle y_{m}\right\rangle$ is a $*$-valuated coproduct with $N / N^{\prime}$ finite.

PROOF. Write $y_{1}=n_{1} x_{1}+n_{2} x_{2}+\cdots+n_{m} x_{m}$ and observe that there is no loss of generality in assuming that each $n_{i}$ is nonzero. But then passing immediately to the *-valuated coproduct $N^{\prime}=\left\langle n_{1} x_{1}\right\rangle \oplus\left\langle n_{2} x_{2}\right\rangle \oplus \cdots \oplus\left\langle n_{m} x_{m}\right\rangle$, we may further assume that $y_{1}=x_{1}+x_{2}+\cdots+x_{m}$. Notice then that we have $\left|y_{1}\right| \leq\left|x_{i}\right|$ for each $i$, and therefore we can rearrange that $x_{i}$ 's so that $x_{1}, \ldots, x_{k}$ have the same type as $y_{1}$ and the remaining $x_{i}$ 's are of strictly larger types. Now write $y_{1}=y+g$ where $y=x_{1}+\cdots+x_{k}$ and $g$ is the sum of the remaining $x_{i}$ 's. Next we make the crucial observation that the primitivity of $y_{1}$ implies that $\left|y_{1}\right|=|y|$. By Lemma 2.7, there are primitive elements $y_{2}, \ldots, y_{k}$ such that $N=\langle y\rangle \oplus\left\langle y_{2}\right\rangle \oplus \cdots \oplus\left\langle y_{k}\right\rangle \oplus\left\langle x_{k+1}\right\rangle \oplus$ $\cdots \oplus\left\langle x_{m}\right\rangle$. Finally, an application of Lemma 2.5 allows us to replace $y$ by $y_{1}$.

COROLlaRY 2.9. If $x$ is a primitive element in the separable group $G$, then $\langle x\rangle_{*}$ is a direct summand of $G$.

Proof. By separability, $x$ is contained in a direct summand $A=A_{1} \oplus A_{2} \oplus \cdots \oplus$ $A_{m}$, where each $A_{i}$ is a rank one subgroup of $G$. Thus we will have $x$ contained in a $*$-valuated coproduct $N=\left\langle x_{1}\right\rangle \oplus\left\langle x_{2}\right\rangle \oplus \cdots \oplus\left\langle x_{m}\right\rangle$, where $x_{i} \in A_{i}$ for each $i$ and $A / N$ is torsion. But then Theorem 2.8 yields a $*$-valuated coproduct $N^{\prime}=$ $\langle x\rangle \oplus\left\langle y_{2}\right\rangle \oplus \cdots \oplus\left\langle y_{m}\right\rangle$, where $N / N^{\prime}$ is finite. Since $B=\langle x\rangle_{*} \oplus\left\langle y_{2}\right\rangle_{*} \oplus \cdots \oplus\left\langle y_{m}\right\rangle_{*}$ is pure in $G$ and $A / N^{\prime}$ is torsion, $B=A$ and $\langle x\rangle_{*}$ is a summand of $G$.

COROLlARY 2.10 (BAER [1]). A finite rank summand of a separable group is completely decomposable.

Proof. Suppose $G=A \oplus K$ is separable and $A$ has finite rank. The proof is by induction on the rank of $A$. Choose $0 \neq x \in A$ to be of maximal type $\sigma$ in $A$. By 2.9 and the implicit induction hypothesis, it suffices to show that $x$ is primitive. Now if $s \in \sigma$ and $g \in G\left(s^{*}\right)$, the choice of $x$ implies that $g \in K$. But then $|x-g|_{p}=|x|_{p} \wedge|g|_{p} \leq|x|_{p}$ for all primes $p$ and hence $x$ is primitive.

Because of their frequent occurrence in the remainder of this paper, we introduce the term free *-valuated subgroup to refer to any subgroup $F$ of $G$ that can be represented as a $*$-valuated coproduct $G=\bigoplus_{i \in I}\left\langle x_{i}\right\rangle$, where the $x_{i}$ 's are nonzero primitive elements of $G$. Under these circumstances, we shall say that the $x_{i}$ 's form a set of free generators of $F$.

THEOREM 2.11. If $F$ and $N$ are free *-valuated subgroups of $G$, where $N$ has finite rank and $N \subseteq F$, then there is a *-valuated coproduct $F^{\prime}=N \oplus M$, where $F / F^{\prime}$ is finite and $M$ is also a free *-valuated subgroup of $G$.

ProOF. Suppose we have $N=\left\langle y_{1}\right\rangle \oplus \cdots \oplus\left\langle y_{m}\right\rangle$, where the $y_{i}$ 's form a set of free generators of $N$. Proceeding by induction, we may assume that we have a *-valuated coproduct $F_{1}=\left\langle y_{1}\right\rangle \oplus \cdots \oplus\left\langle y_{n-1}\right\rangle \oplus M_{1}$, where $F / F_{1}$ is finite and $M_{1}$ 
is a free *-valuated subgroup of $G$. Then some nonzero multiple $y_{n}^{\prime}$ of $y_{n}$ is in $F_{1}$. Let $s=\left|y_{n}^{\prime}\right|$. Now we can write $y_{1}^{\prime}+\cdots+y_{n-1}^{\prime}+y_{n}^{\prime}=y+g$ where the $y_{i}^{\prime}$ 's are multiples of the $y_{i}$ 's, $g \in M_{1} \cap G\left(s^{*}\right)$ and $y$ is a primitive element in $M_{1}$ having the same type as $y_{n}^{\prime}$. Since $y_{n}^{\prime}$ is primitive and $\left\langle y_{1}\right\rangle \oplus \cdots \oplus\left\langle y_{n}\right\rangle$ is a $*$-valuated coproduct, it follows that $|y|=\left|y_{n}^{\prime}\right|$. Then, just as in the proof of 2.8 , we have a *-valuated coproduct $M_{1}^{\prime}=\langle y\rangle \oplus M$, where $M_{1} / M_{1}^{\prime}$ is finite and $M$ is a free *-valuated subgroup containing $g$. Using Lemma 2.5, we conclude that we have a *-valuated coproduct $F_{1}^{\prime}=\left\langle y_{1}\right\rangle \oplus \cdots \oplus\left\langle y_{n-1}\right\rangle \oplus\left\langle y_{n}^{\prime}\right\rangle \oplus M$ with $F / F_{1}^{\prime}$ finite. Since $y_{n}$ has finite order modulo $\left\langle y_{n}^{\prime}\right\rangle$, we still have a $*$-valuated coproduct when $\left\langle y_{n}^{\prime}\right\rangle$ is replaced by $\left\langle y_{n}\right\rangle$, yielding thereby the desired $F^{\prime}$.

COROLlARY 2.12. If $N$ is a finite rank, free *-valuated subgroup of the separable group $G$, then the pure closure of $N$ is a direct summand of $G$.

ProOF. Let $N=\left\langle y_{1}\right\rangle \oplus \cdots \oplus\left\langle y_{n}\right\rangle$, where the $y_{i}$ 's are free generators of $N$. Since $G$ is separable, $N$ is contained in a direct summand $A=A_{1} \oplus \cdots \oplus A_{m}$ of $G$, where each $A_{i}$ is a rank one subgroup. Each $A_{i}$ is locally cyclic and therefore we have nonzero $x_{i}$ 's in the corresponding $A_{i}$ 's such that $F=\left\langle x_{1}\right\rangle \oplus \cdots \oplus\left\langle x_{n}\right\rangle$ contains $N$. By 2.11 , we have a $*$-valuated coproduct $F^{\prime}=N \oplus M$, where $F / F^{\prime}$ is finite. Thus $A$ is the pure closure of $F^{\prime}$ and we clearly have a direct decomposition $A=B \oplus C$, where $B$ and $C$ are, respectively, the pure closures of $N$ and $M$ in $G$.

COROLlARY 2.13 (BAER [1]). Any finite rank, pure subgroup of a homogeneous separable group is a direct summand.

ProOF. Let $A$ be a nonzero finite rank pure subgroup of the homogeneous separable group $G$ and let $N$ be a free subgroup of $A$ with $A / N$ torsion. As in the proof of 2.12, $N$ is contained in a finite rank, free *-valuated subgroup $F$ of $G$. Now notice that Lemma 2.7 implies that all the elements of $N$ are primitive in $G$. By Theorem 2.8, we have *-valuated coproduct $F^{\prime}=\left\langle y_{1}\right\rangle \oplus \cdots \oplus\left\langle y_{n}\right\rangle$, where $F / F^{\prime}$ is finite, the $y_{i}$ 's are primitive in $G$ and $y_{1}$ is a nonzero element of $N$. A straightforward induction then leads to a free *-valuated subgroup $N^{\prime}$ of $G$ with $N / N^{\prime}$ finite. Since $A$ is the pure closure of $N^{\prime}$, the desired conclusion follows from 2.12 .

3. $k$-groups. In this section, we study a class of torsion free groups more general than the separable groups. We call $G$ a $k$-group if each finite subset can be imbedded in a finite rank, free $*$-valuated subgroup.

EXAMPLE 3.1. A $k$-group need not be separable. Let $K=Z^{\aleph_{0}}$ and take $H$ to be the corresponding direct sum of $\aleph_{0}$ copies of $Z$. Then for any prime $p, G=H+p K$ is an $\aleph_{1}$-free group that is not separable (see p. 114 of [4]). On the other hand, $G$ is a $k$-group since it is $\aleph_{1}$-free. Indeed, any finite subset is imbeddable in a finite rank, pure free subgroup $F=\left\langle x_{1}\right\rangle \oplus\left\langle x_{2}\right\rangle \oplus \cdots \oplus\left\langle x_{n}\right\rangle$. As $F$ is pure, this direct decomposition is a valuated coproduct. Furthermore $G\left(s^{*}\right)=0$ for all height sequences $s$ since $G$ is homogeneous of type $(0,0, \ldots, 0, \ldots)$. Consequently, each $x_{i}$ is primitive and $F=\left\langle x_{1}\right\rangle \oplus\left\langle x_{2}\right\rangle \oplus \cdots \oplus\left\langle x_{n}\right\rangle$ is a $*$-valuated coproduct in $G$.

Example 3.1 notwithstanding, we can prove the following result.

Proposition 3.2. A finite rank summand of a $k$-group is completely decomposable. 
Proof. Let $A$ be a finite rank summand of the $k$-group $G$. Then there is a finitely generated subgroup $F$ of $A$ with $A / F$ torsion. Since $G$ is a $k$-group, $F$ is contained in a free *-valuated subgroup $N$ of $G$. The pure closure $B$ of the subgroup $N$ is a completely decomposable group containing $A$. But then $A$ is a direct summand of $B$ and therefore $A$ is completely decomposable by 2.10 .

Separable groups have received much attention in the literature. The following result provides a useful characterization of these groups.

THEOREM 3.3. $G$ is separable if and only if $G$ is a $k$-group with the property that the pure closure of each finite rank, free *-valuated subgroup is a direct summand.

PROOF. Since separable groups are obviously $k$-groups, the proposition is an immediate consequence of 2.12 and 3.2.

Like separable groups, countable $k$-groups are completely decomposable. But the proof of this fact requires the following lemma.

LEMMA 3.4. Let $N$ be a finite rank, free *-valuated subgroup of the $k$-group $G$ and suppose $S$ is a finite subset of $G$. Then there exists a finite collection of primitive elements $y_{1}, y_{2}, \ldots, y_{m}$ such that $N^{\prime}=N \oplus\left\langle y_{1}\right\rangle \oplus\left\langle y_{2}\right\rangle \oplus \cdots \oplus\left\langle y_{m}\right\rangle$ is a *-valuated coproduct in $G$ with $\left\langle S, N^{\prime}\right\rangle / N^{\prime}$ finite.

PROOF. Let $x_{1}, x_{2}, \ldots, x_{n}$ be free generators of $N$ and take $S^{\prime}=S \cup\left\{x_{1}, x_{2}, \ldots\right.$, $\left.x_{n}\right\}$. Since $G$ is a $k$-group, we can select a finite rank, free *-valuated subgroup $F$ containing $S^{\prime}$. Then $N \subseteq F$ and, by Theorem 2.11 , we have a $*$-valuated coproduct $N^{\prime}=N \oplus\left\langle y_{1}\right\rangle \oplus\left\langle y_{2}\right\rangle \oplus \cdots \oplus\left\langle y_{m}\right\rangle$, where the $y_{i}$ 's are primitive and $F / N^{\prime}$ is finite. Since $S \subseteq F$, the proof is complete.

THEOREM 3.5. A countable $k$-group is completely decomposable.

ProOF. Suppose $x_{1}, x_{2}, \ldots, x_{n}, \ldots$ is an enumeration of the elements of $G$ and let $X_{n}=\left\{x_{i}: i<n\right\}$ for each $n<\omega$. Using 3.4, we define inductively an ascending sequence $\left\{S_{n}\right\}$, where each $S_{n}$ is a finite set of primitive elements serving as a free basis of a free *-valuated subgroup $F_{n}$ with $\left\langle X_{n}, F_{n}\right\rangle / F_{n}$ finite. Then $F=\bigcup_{n<\omega} F_{n}$ is a free *-valuated subgroup of $G$ with $G / F$ torsion. Thus $G$ is the pure closure of $F$ and therefore $G$ is completely decomposable.

We next want to show that the class of $k$-groups is closed under the operation of taking direct summands. Our proof of this fact requires a technical lemma, which also turns out to be useful later. The statement of this lemma, however, requires a couple of preliminary definitions. If $H \oplus K$ is a $*$-valuated coproduct in $G$ and if $F$ is a subgroup of $G$, then we say that $F$ splits along $H$ and $K$ provided $F=(F \cap H) \oplus(F \cap K)$ and we say that $F$ is quasi-splitting along $H$ and $K$ if $F /(F \cap H) \oplus(F \cap K)$ is torsion.

LEMMA 3.6. Suppose $H \oplus K$ is a *-valuated coproduct in $G$ and let $F$ be a free *-valuated subgroup that is quasi-splitting along $H$ and $K$. If the finite rank, free *-valuated subgroup $A$ is contained in $F$, then there is a *-valuated coproduct $F^{\prime}=A \oplus B \oplus C$, where $F / F^{\prime}$ is torsion, $B$ has finite rank and $C$ splits along $H$ and $K$.

ProOF. The proof is by induction on the rank of $A$ and we impose the further hypothesis that $B$ be a free *-valuated subgroup having a set of free generators each 
of which has the same type as one of the members of a fixed set of free generators of $A$. The proof also requires the following sublemma: If $N$ is quasi-splitting along $H$ and $K$ and if $N^{\prime}=M \oplus L$ is a $*$-valuated coproduct, where $N / N^{\prime}$ is torsion and $M$ splits along $H$ and $K$, then there exists a $*$-valuated coproduct $N^{\prime \prime}=M \oplus L^{\prime}$, where $N / N^{\prime \prime}$ is torsion and $L^{\prime}$ also splits along $H$ and $K$. Indeed we need only take $N^{\prime \prime}=N^{\prime} \cap H+N^{\prime} \cap K$ and $L^{\prime}=H_{1} \oplus K_{1}$, where $H_{1}=H \cap[(M \cap K) \oplus L]$ and $K_{1}=K \cap[(M \cap H) \oplus L]$.

Suppose $A=A_{1} \oplus\langle x\rangle$ is a $*$-valuated coproduct where $x$ is a primitive element of maximal type in $A$. By our induction hypothesis, we have a $*$-valuated coproduct $F_{1}=A_{1} \oplus B_{1} \oplus C_{1}$, where $F / F_{1}$ is torsion, $B_{1}$ satisfies the appropriate conditions and $C_{1}$ splits along $H$ and $K$. Notice that $F$ being quasi-splitting along $H$ and $K$ is essential here even in the case $A_{1}=0$. Then some nonzero multiple $x^{\prime}$ of $x$ lies in $F_{1}$ and we write $x^{\prime}=a+b+c_{1}+c_{2}$, where $a \in A_{1}, b \in B_{1}, c_{1} \in C_{1} \cap H$ and $c_{2} \in C_{1} \cap K$. Let $s=\left|x^{\prime}\right|$. Since $\left|x^{\prime}\right| \leq|a|$, either $a=0$ or else $a$ has maximal type in $A_{1}$. Thus Lemma 2.7 implies that $a$ and $x^{\prime}-a=b+c_{1}+c_{2}$ are primitive elements. Moreover, since $A_{1} \oplus\left\langle x^{\prime}\right\rangle$ is a valuated coproduct and $\left|x^{\prime}\right| \leq\left|b+c_{1}+c_{2}\right|$, it follows that $\left|b+c_{1}+c_{2}\right|=s$. Even though $c_{1}$ and $c_{2}$ need not themselves be primitive elements, there are, we claim, elements $z_{1} \in H \cap G\left(s^{*}\right)$ and $z_{2} \in K \cap G\left(s^{*}\right)$ such that $h=c_{1}-z_{1}$ and $k=c_{2}-z_{2}$ are primitive. As the arguments are parallel, we provide the details only for the choice of $z_{1}$. If $c_{1} \in G\left(s^{*}\right)$, then we need only take $z_{1}=c_{1}$. Assume then that $c_{1} \notin G\left(s^{*}\right)$. As $c_{1}$ is contained in the free $*$-valuated subgroup $F$ and $s \leq\left|c_{1}\right|$, we can write $c_{1}=v+w$, where $w \in G\left(s^{*}\right) \cap F$ and $v$ is a primitive element having the same type as $c_{1}$. Moreover, since $F$ is quasi-splitting along $H$ and $K$, some nonzero multiple of $w$ is expressible as a sum $z_{1}+w^{\prime}$, where $z_{1} \in F \cap H$ and $w^{\prime} \in F \cap K$. Replacing $x^{\prime}$ by some nonzero multiple of itself if necessary, we may assume that $c_{1}=v+z_{1}+w^{\prime}$ and that also $z_{1} \in F_{1}$. Notice then that $z_{1} \in G\left(s^{*}\right)$ since $H \oplus K$ is a $*$-valuated coproduct. Then $h=c_{1}-z_{1}=v+w^{\prime}$ is primitive by Lemma 2.3 because $|v|=|h| \wedge\left|w^{\prime}\right|$ since $h \in H$ and $w^{\prime} \in K$.

Observe that the choice of $x$ and the condition on $B_{1}$ force $F_{1} \cap G\left(s^{*}\right) \subseteq C_{1}$. Thus from our choice of $z_{1}$ above, $h \in C_{1}$ and, similarly, $k=c_{2}-z_{2} \in C_{1}$. By Theorem 2.11 and the fact that $C_{1}$ is contained in the free *-valuated subgroup $F$, we have a *valuated coproduct $C_{1}^{\prime}=\langle h\rangle \oplus\langle k\rangle \oplus C$, where $C_{1} / C_{1}^{\prime}$ is torsion and $C_{1} \cap G\left(s^{*}\right) \subseteq C$. By our sublemma above, we may further assume that $C$ splits along $H$ and $K$. Now clearly $F_{2}=A_{1} \oplus B_{1} \oplus\langle h\rangle \oplus\langle k\rangle \oplus C$ is a $*$-valuated coproduct with $F / F_{2}$ torsion. Let $z=x^{\prime}-z_{1}-z_{2}=a+b+h+k$ and note that $z$ is a primitive element with $|z|=s$ by Lemma 2.3. Also $y_{1}=b+h+k$ is primitive with $\left|y_{1}\right|=\left|b+c_{1}+c_{2}\right|=s$ for the same reason. Since $\left|x^{\prime}\right| \leq|b|$, either $b=0$ or $b$ is a primitive element in $B_{1}$ of maximal type. In either case, we have a $*$-valuated coproduct $B_{1}^{\prime}=B_{0} \oplus\langle b\rangle$, where $B_{1} / B_{1}^{\prime}$ is finite and $B_{0}$ is a free *-valuated subgroup of $G$. Consider then the *-valuated coproduct $F^{\prime}=A_{1} \oplus B_{0} \oplus\langle b\rangle \oplus\langle h\rangle \oplus\langle k\rangle \oplus C$ and apply Lemma 2.7 to express $F^{\prime}$ as the $*$-valuated coproduct $F^{\prime}=A_{1} \oplus B_{0} \oplus\left\langle y_{1}\right\rangle \oplus\left\langle y_{2}\right\rangle \oplus\left\langle y_{3}\right\rangle \oplus C$, where $y_{2}$ and $y_{3}$ are also primitive. Lemma 2.5 allows us to replace $y_{1}$ by $z$, and since $z_{1}, z_{2} \in C$ another application of Lemma 2.5 enables us to replace $z$ by $x^{\prime}$. Thus we have a $*$-valuated coproduct $F^{\prime}=A_{1} \oplus\left\langle x^{\prime}\right\rangle \oplus B \oplus C$, where $B=B_{0} \oplus\left\langle y_{2}\right\rangle \oplus\left\langle y_{3}\right\rangle$, $F / F^{\prime}$ is torsion and $C$ splits along $H$ and $K$. Finally, as $x$ has finite order modulo $\left\langle x^{\prime}\right\rangle$, we may replace $A_{1} \oplus\left\langle x^{\prime}\right\rangle$ by $A=A_{1} \oplus\langle x\rangle$.

THEOREM 3.7. A direct summand of a $k$-group is a $k$-group. 
Proof. Suppose $G=H \oplus K$ is a $k$-group and let $S$ be a finite subset of $H$. Then $S \subseteq A$ for some finite rank, free *-valuated subgroup $A$ of $G$. Using 3.4, we define inductively a pair of ascending sequences $\left\{T_{n}\right\}_{n<\omega}$ and $\left\{S_{n}\right\}_{n<\omega}$ of finite subsets of $G$ satisfying the following conditions:

(i) $T_{n}$ is a set of free generators of a free *-valuated subgroup $F_{n}$ of $G$ with $F_{0}=A$.

(ii) $F_{n} \subseteq\left\langle S_{n}\right\rangle$ and $S_{n}=\left(S_{n} \cap H\right) \cup\left(S_{n} \cap K\right)$.

(iii) $\left\langle S_{n}, F_{n+1}\right\rangle / F_{n+1}$ is finite for each $n$.

Then $F=\bigcup_{n<\omega} F_{n}$ is a free *-valuated subgroup of $G$, and conditions (ii) and (iii) imply that $F$ is quasi-splitting along $H$ and $K$. Then we have a $*$-valuated coproduct $F^{\prime}=A \oplus B \oplus C$, where $F^{\prime}, B$ and $C$ satisfy the conditions stated in Lemma 3.6. Recalling the sublemma used in the proof of 3.6 , we see that there is a $*$-valuated coproduct $F^{\prime \prime}=A_{1} \oplus B_{1} \oplus C$, where $F / F^{\prime \prime}$ is torsion and $A_{1}=$ $H \cap[A \oplus B \oplus(C \cap K)]$. Notice that $A_{1}$ contains $S$ and that $A_{1}$ is necessarily a finite rank subgroup of $G$. Now let $L$ and $M$ be the pure closures of $A_{1}$ and $F$, respectively. Then $M$ is completely decomposable and, since $M$ is also the pure closure of $F^{\prime \prime}, L$ is a direct summand of $M$. By $2.10, L$ is completely decomposable and clearly then $S$ is contained in a direct sum $\left\langle y_{1}\right\rangle \oplus\left\langle y_{2}\right\rangle \oplus \cdots \oplus\left\langle y_{m}\right\rangle$, where the $y_{i}$ 's are primitive in $M$ and this direct sum is a $*$-valuated coproduct in $M$. That the $y_{i}$ 's are actually primitive in $G$ and that $\left\langle y_{1}\right\rangle \oplus\left\langle y_{2}\right\rangle \oplus \cdots \oplus\left\langle y_{m}\right\rangle$ is a *valuated coproduct in $G$ follow from the crucial fact that $M \cap G\left(s^{*}, p\right)=M\left(s^{*}, p\right)$ for all height sequences $s$ and all primes $p$. Indeed from the construction of $M$, $M=\bigoplus_{i<\omega} M_{i}$ is a $*$-valuated coproduct in $G$ where each $M_{i}$ is the pure subgroup of $G$ generated by some primitive element $x_{i}$ of $G$. Thus $M \cap G\left(s^{*}, p\right)=M\left(s^{*}, p\right)$ reduces to $M_{i} \cap G\left(S^{*}, p\right)=M_{i}\left(s^{*}, p\right)$ for each $i$, and this latter fact is an easy consequence of the fact that $M_{i}$ is pure in $G$ and each element of $M_{i}$ is primitive in $G$.

COROLLARY 3.8 (FUCHS [2]). A direct summand of a separable group is separable.

Proof. Suppose $G=H \oplus K$ is separable. Then $H$ is a $k$-group by Theorem 3.7. Clearly $H \cap G\left(s^{*}\right)=H\left(s^{*}\right)$ and $H \cap G\left(s^{*}, p\right)=H\left(s^{*}, p\right)$ for all height sequences $s$ and all primes $p$. The latter observation implies that each free $*$-valuated subgroup of $H$ is a free *-valuated subgroup of $G$. That $H$ is separable follows now from Proposition 3.3.

4. Knice subgroups. In this section, we introduce a class of subgroups that plays a role for torsion free groups analogous to that played by nice subgroups in the study of torsion groups. Indeed, following the theme of our earlier paper [8], we elevate to the status of a definition the property exhibited in 3.4 of finite rank, free $*$-valuated subgroups of $k$-groups.

DEFinition 4.1. A subgroup $N$ of the torsion free group $G$ is said to be a knice subgroup if for each finite subset $S$ of $G$ there exist primitive elements $y_{1}, y_{2}, \ldots, y_{m}$ such that $N^{\prime}=N \oplus\left\langle y_{1}\right\rangle \oplus\left\langle y_{2}\right\rangle \cdots\left\langle y_{m}\right\rangle$ is a $*$-valuated coproduct with $\left\langle S, N^{\prime}\right\rangle / N^{\prime}$ finite.

It is easy to see that $N$ is knice in $G$ if and only if its pure closure is knice in $G$. If $N$ is both pure and knice in $G$, then a routine argument shows that the $y_{i}$ 's can be chosen so that $S \subseteq N^{\prime}$. Note then that $G$ is a $k$-group if and only if 0 is 
a knice subgroup of $G$. That summands of $k$-groups are knice is a nontrivial fact that follows from Theorem 3.7.

PROPOSITION 4.2. If $N^{\prime}=N \oplus\left\langle x_{1}\right\rangle \oplus\left\langle x_{2}\right\rangle \oplus \cdots \oplus\left\langle x_{n}\right\rangle$ is a *-valuated coproduct in $G$ with $N$ a knice subgroup of $G$ and each $x_{i}$ primitive in $G$, then $N^{\prime}$ is a knice subgroup of $G$.

PROOF. By induction, it suffices to consider the case $n=1$. Assume then that $N^{\prime}=N \oplus\langle x\rangle$ is a $*$-valuated coproduct in $G$ with $N$ knice and $x$ primitive. Let $S$ be a finite subset of $G$ and take $S^{\prime}=S \cup\{x\}$. Since $N$ is knice in $G$, there is a *-valuated coproduct $F=N \oplus\left\langle y_{1}\right\rangle \oplus\left\langle y_{2}\right\rangle \oplus \cdots \oplus\left\langle y_{m}\right\rangle$, where the $y_{i}$ 's are primitive and $\left\langle S^{\prime}, F\right\rangle / F$ is finite. In particular, some nonzero multiple $x^{\prime}$ of $x$ is contained in $F$. Thus we can write $x^{\prime}=z+t_{1} y_{1}+t_{2} y_{2}+\cdots+t_{m} y_{m}$, where $z \in N$. Since $N \oplus\left\langle x^{\prime}\right\rangle$ is a $*$-valuated coproduct, if all the $t_{i} y_{i}$ 's had type greater than the type of $x^{\prime}$, the primitivity of $x^{\prime}$ would be contradicted. Then $x^{\prime}=z+y+g$ where the primitive element $y$ is the sum of the $t_{i} y_{i}$ 's having the same type as $x^{\prime}$. Observe that $x^{\prime}-z=y+g$ is also primitive since if $x^{\prime}-z \in G\left(t^{*}, p\right)$ where $t$ is equivalent to $\left|x^{\prime}-z\right|=\left|x^{\prime}\right| \wedge|z|=\left|x^{\prime}\right|$ and $t_{p}=\left|x^{\prime}-z\right|_{p}=\left|x^{\prime}\right|_{p} \neq \infty$, then $N \oplus\left\langle x^{\prime}\right\rangle$ being a *-valuated coproduct implies $x^{\prime} \in G\left(t^{*}, p\right)$ contrary to the fact that $x^{\prime}$ is primitive. But then $g \in G\left(s^{*}\right)$, where $s=\left|x^{\prime}\right|=\left|x^{\prime}-z\right|$ and hence the equation $x^{\prime}-z=y+g$ forces us to conclude that $|y|=\left|x^{\prime}-z\right|=\left|x^{\prime}\right|$. By Lemma 2.7, $x^{\prime}$ is contained in a *-valuated coproduct $F^{\prime}=N \oplus\langle y\rangle \oplus\left\langle z_{2}\right\rangle \oplus \cdots \oplus\left\langle z_{m}\right\rangle$ where $F / F^{\prime}$ is finite and, of course, the $z_{i}$ 's are primitive. Lemma 2.5 then allows us to replace $y$ by $x^{\prime}$ in the representation of $F^{\prime}$ above. Finally, we note that $H=N \oplus\langle x\rangle \oplus\left\langle z_{2}\right\rangle \oplus \cdots \oplus\left\langle z_{m}\right\rangle$ is a *-valuated coproduct with $\langle S, H\rangle / H$ finite, that is, $N \oplus\langle x\rangle$ is a knice subgroup of $G$.

Recall that a pure subgroup $H$ of the torsion free group $G$ is said to be balanced if each coset $x+H$ contains an element $y$ such that $|y|=|x+H|$, where the height sequences are computed in $G$ and $G / H$, respectively.

THEOREM 4.3. A pure subgroup $H$ of $G$ is a knice subgroup if and only if $H$ is balanced in $G$ and $G / H$ is a k-group.

PROOF. First assume that $H$ is a knice and pure subgroup of $G$. Then for any $x$ in $G$ we can write $x=z+y$, where $z \in H$ and $H \oplus\langle y\rangle$ is a valuated coproduct in $G$. It follows that $|x+h| \leq|y|$ for all $h \in H$, that is, $|x+H|=|y|$ and $H$ is balanced. Since $H$ is balanced in $G,(G / H)(s)=G(s)+H / H$ and $(G / H)\left(s^{*}, p\right)=G\left(s^{*}, p\right)+H / H$ for all height sequences $s$ and all primes $p$. To prove that $G / H$ is a $k$-group it is clearly enough to show that if $H \oplus\left\langle y_{1}\right\rangle \oplus \cdots \oplus\left\langle y_{m}\right\rangle$ is a *-valuated coproduct in $G$ with primitive $y_{i}$ 's, then the $y_{i}+H$ 's are primitive and $\left\langle y_{1}+H\right\rangle \oplus \cdots \oplus\left\langle y_{m}+H\right\rangle$ is a $*$-valuated coproduct in $G / H$. But these facts are easily established using the observations above about $(G / H)(s)$ and $(G / H)\left(s^{*}, p\right)$.

Conversely, assume that $H$ is balanced in $G$ and that $G / H$ is a $k$-group. Now consider any $*$-valuated coproduct $\left\langle y_{1}+H\right\rangle \oplus \cdots \oplus\left\langle y_{m}+H\right\rangle$ in $G / H$, where the $y_{i}+H$ 's are primitive and $\left|y_{i}\right|=\left|y_{i}+H\right|$ for each $i$. It is trivial then that the $y_{i}$ 's are primitive in $G$, and obviously it is enough to argue that $H \oplus\left\langle y_{1}\right\rangle \oplus \cdots \oplus\left\langle y_{m}\right\rangle$ is a $*$-valuated coproduct in $G$. Let $x=h+t_{1} y_{1}+\cdots+t_{m} y_{m}$, where $h \in H$. First suppose $x \in G(s)$. Then, for each $i, t_{i} y_{i}+H \in(G / H)(s)$ and hence $t_{i} y_{i} \in G(s)$ since $\left|t_{i} y_{i}\right|=\left|t_{i} y_{i}+H\right|$. Thus $H \oplus\left\langle y_{1}\right\rangle \oplus \cdots \oplus\left\langle y_{m}\right\rangle$ is at least a valuated coproduct. Next suppose $x \in G\left(s^{*}, p\right)$. Then once again for each $i, t_{i} y_{i}+H \in(G / H)\left(s^{*}, p\right)$. 
But since $t_{i} y_{i}+H$ is primitive, either $t_{i} y_{i}+H$ is in $(G / H)(p s)$ of $\left|t_{i} y_{i}+H\right|$ is not equivalent to $s$. In either case, $\left|t_{i} y_{i}\right|=\left|t_{i} y_{i}+H\right|$ implies $t_{i} y_{i} \in G\left(s^{*}, p\right)$.

Since the ideas involved in the proof of Theorem 3.4 can be used to show that every countable subset of a $k$-group is contained in a countable completely decomposable pure subgroup, the following corollary implies that pure knice subgroups are $\aleph_{1}$-pure.

COROLLARY 4.4. If $H$ is a pure knice subgroup of $G$ and if $G / H$ is countable, then $H$ is a direct summand of $G$.

Proof. As is well known, $H$ is a summand of $G$ if $H$ is balanced in $G$ and $G / H$ is completely decomposable. Thus the result follows from 4.3 and 3.5 .

COROLLARY 4.5. If $H$ is balanced in $G$ and $K / H$ is a pure knice subgroup of $G / H$, then $K$ is a pure knice subgroup of $G$.

Proof. If $H$ is balanced in $G$ and $K / H$ is balanced in $G / H$, then $K$ is balanced in $G$. Thus Theorem 4.3 and the canonical isomorphism $G / K \simeq(G / H) /(K / H)$ yield the desired conclusion.

COROLlaRY 4.6. Let $H$ be a pure subgroup of $G$. If $H$ is knice in $G$ and $N / H$ is knice in $G / H$, then $N$ is knice in $G$.

ProOF. It suffices to observe that the hypotheses imply that the pure closure $K$ of $N$ is a knice subgroup of $G$. But since $K / H$ is the pure closure in $G / H$ of the knice subgroup $N / H$, we conclude that $K$ is knice in $G$ by 4.5 .

Formalizing a notion that has already played a role in 3.7 and 3.8 , we say that a pure subgroup $H$ of $G$ is *-pure if $H \cap G\left(s^{*}\right)=H\left(s^{*}\right)$ and $H \cap G\left(s^{*}, p\right)=H\left(s^{*}, p\right)$ for all height sequences $s$ and all primes $p$. We have already seen that summands are *-pure subgroups, and so are rank one pure subgroups generated by primitive elements. Clearly the ascending union of $*$-pure subgroups is *-pure. Also a *valuated coproduct $H=\bigoplus_{i \in I} H_{i}$ in $G$ will be a $*$-pure subgroup if each $H_{i}$ is *-pure in $G$.

Proposition 4.7. A pure knice subgroup is *-pure.

ProOF. Suppose $H$ is a pure knice subgroup of $G$ and let $x \in H \cap G\left(s^{*}, p\right)$. Then we can write $x=z_{1}+z_{2}+\cdots+z_{n}+g$, where $g \in G(p s)$ and each $z_{i}$ is an element of $G(s)$ with $\left|z_{i}\right|$ not equivalent to $s$. Since $H$ is a pure knice subgroup of $G,\left\{z_{1}, z_{2}, \ldots, z_{n}, g\right\}$ is contained in a $*$-valuated coproduct $H \oplus\left\langle y_{1}\right\rangle \oplus \cdots \oplus\left\langle y_{m}\right\rangle$, where the $y_{i}$ 's are primitive in $G$. Then we can write $g=h+t_{1} y_{1}+\cdots+t_{m} y_{m}$ and, for each $i, z_{i}=h_{i}+t_{1}^{(i)} y_{1}+\cdots+t_{m}^{(i)} y_{m}$ where $h$ and each $h_{i}$ is in $H$. Since $|g| \leq|h|$ and $\left|z_{i}\right| \leq\left|h_{i}\right|$ for $i=1,2, \ldots, n, x=z_{1}+\cdots+z_{m}+g=h_{1}+h_{2}+\cdots+h_{n}+h$ is in $H\left(s^{*}, p\right)$. Likewise, if $x \in H \cap G\left(s^{*}\right)$, then clearly $x \in H\left(s^{*}\right)$.

Exploiting Lemma 3.6 again, we shall prove that a pure knice subgroup of a $k$-group is itself a $k$-group.

THEOREM 4.8. The pure knice subgroup $H$ of $G$ is a $k$-group if and only if $G$ is a k-group.

PROOF. First consider the case where $H$ is a $k$-group and let $S$ be a finite subset of $G$. Since $H$ is a pure knice subgroup of $G, S$ is contained in a $*$-valuated 
coproduct $H \oplus\left\langle y_{1}\right\rangle \oplus \cdots \oplus\left\langle y_{m}\right\rangle$, where the $y_{i}$ 's are primitive in $G$. But then $S \subseteq\langle T\rangle \oplus\left\langle y_{1}\right\rangle \oplus \cdots \oplus\left\langle y_{m}\right\rangle$ for some finite subset $T$ of $H$. Since $H$ is a $k$-group, there are primitive elements $x_{1}, \ldots, x_{n}$ in $H$ such that $\left\langle x_{1}\right\rangle \oplus \cdots \oplus\left\langle x_{n}\right\rangle$ is a *valuated coproduct in $H$ containing $T$. Then 4.7 implies that $F=\left\langle x_{1}\right\rangle \oplus \cdots \oplus$ $\left\langle x_{n}\right\rangle \oplus\left\langle y_{1}\right\rangle \oplus \cdots \oplus\left\langle y_{m}\right\rangle$ is a free *-valuated subgroup of $G$ that contains $S$.

Conversely, assume that $G$ is a $k$-group. The proof that $H$ is a $k$-group will involve an elaboration on the technique used to prove Theorem 3.7 , and we shall need to use Proposition 4.2 in exploiting the fact that $H$ is knice in order to generate an appropriate $K$ with $H \oplus K$ a *-valuated coproduct in $G$. Once again we begin with a finite rank, free *-valuated subgroup $A$ of $G$ which contains some fixed finite subset $S$ of $H$. But this time we define inductively, in addition to a pair of ascending sequences $\left\{T_{n}\right\}_{n<\omega}$ and $\left\{S_{n}\right\}_{n<\omega}$ of finite subsets of $G$, a third sequence $\left\{B_{n}\right\}_{n<\omega}$ of finite rank, pure knice subgroups such that the following conditions are satisfied:

(i) $T_{n}$ is a set of free generators of a free *-valuated subgroup $F_{n}$ of $G$ with $F_{0}=A$.

(ii) $H \oplus B_{0} \oplus \cdots \oplus B_{n}$ is a $*$-valuated coproduct in $G$ that contains $F_{n}$.

(iii) $F_{n} \subseteq\left\langle S_{n}\right\rangle$ and $S_{n}=\left(S_{n} \cap H\right) \cup\left(S_{n} \cap\left(B_{0} \oplus \cdots \oplus B_{n}\right)\right)$.

(iv) $\left\langle S_{n}, F_{n+1}\right\rangle / F_{n+1}$ is finite for each $n$.

Then $F=\bigcup_{n<\omega} F_{n}$ is a free *-valuated subgroup of $G$ that is quasi-splitting along $H$ and $K=\bigoplus_{n<\omega} B_{n}$. The proof of the theorem is now completed exactly as in the case of Theorem 3.7.

The preceding theorem leads quickly to a generalization of the important observation of Fuchs that summands of separable groups are separable.

COROLLARY 4.9. A pure knice subgroup of a separable group is itself separable.

ProOF. Suppose $H$ is a pure knice subgroup of the separable group $G$. Since $H$ is *-pure in $G$ by 4.7 , each free *-valuated subgroup of $H$ is a free *-valuated subgroup of $G$. That $H$ is separable then follows from 4.8 and 3.3.

In [9], Rangaswamy introduces the notion of a strongly balanced subgroup and his Theorem 7 asserts that both $H$ and $G / H$ are separable if $H$ is strongly balanced in the separable group $G$. Thus strongly balanced subgroups of separable groups are actually pure knice subgroups and hence 4.9 can also be viewed as a partial generalization of Rangaswamy's theorem. Notice, however, that in the context of separable groups, the pure knice subgroups form a more comprehensive class than the strongly balanced subgroups. Indeed if $H$ is a pure knice subgroup of the separable groups $G$, then $G / H$ need not necessarily be separable. This latter observation follows from $3.1,4.3$ and the well-known fact that every torsion free group is a homomorphic image of a completely decomposable group with balanced kernel.

We close this section by recording some further facts about pure knice subgroups.

PROPOSITION 4.10. Let $H$ and $K$ be pure subgroups of the torsion free group $G$ with $H \subseteq K$.

(i) If $H$ is knice in $K$ and $K$ is knice in $G$, then $H$ is knice in $G$.

(ii) If $H$ is knice in $G$ and $K / H$ is knice in $G / H$, then $K$ is knice in $G$.

(iii) If $K$ is knice in $G$, then $K / H$ is knice in $G / H$.

(iv) If $H$ and $K$ are both knice in $G$, then $H$ is knice in $K$. 
ProOF. Of course, (ii) has already been proved in 4.6. To prove the transitivity result (i), we take $S$ to be a finite subset of $G$. Since we are assuming that $K$ is pure and knice in $G$, we have $S$ contained in a *-valuated coproduct $K \oplus\left\langle y_{1}\right\rangle \oplus$ $\left\langle y_{2}\right\rangle \oplus \cdots \oplus\left\langle y_{m}\right\rangle$, where the $y_{i}$ 's are primitive in $G$. But then $S$ has a corresponding finite projection $T$ in $K$, that is, $S \subseteq\left\langle T, y_{1}, y_{2}, \ldots, y_{m}\right\rangle$, where $T$ is a finite subset of $K$. But because $H$ is assumed to be knice in $K, T$ is contained in a $*$-valuated coproduct (in $K$ ) $H \oplus\left\langle x_{1}\right\rangle \oplus\left\langle x_{2}\right\rangle \oplus \cdots \oplus\left\langle x_{n}\right\rangle$, where $x_{j}$ 's are primitive in $K$. By 4.7, the $x_{j}$ 's are primitive in $G$ and $H \oplus\left\langle x_{1}\right\rangle \oplus \cdots \oplus\left\langle x_{n}\right\rangle \oplus\left\langle y_{1}\right\rangle \oplus \cdots \oplus\left\langle y_{m}\right\rangle$ is a *-valuated coproduct in $G$ containing $S$; that is, $H$ is knice in $G$.

That $K$ being knice in $G$ implies $K / H$ is knice in $G / H$ is an easy consequence of Theorem 4.3. Indeed under these circumstances, $K / H$ is clearly balanced in $G / H$ and $(G / H) /(K / H) \simeq G / K$ is a $k$-group. Finally, to prove (iv), we assume that both $H$ and $K$ are knice in $G$. Then $G / H$ is a $k$-group by 4.3 , and therefore (iii) and Theorem $4.8 \mathrm{imply}$ that $K / H$ is a $k$-group. Noting that $H$ is balanced in $K$ since it is balanced in $G$ by 4.3 , we see that yet another application of 4.3 yields that desired conclusion that $H$ is knice in $K$.

COROLlaRY 4.11. If $H=A \oplus B$ is a pure knice subgroup of the k-group $G$, then $A$ and $B$ are pure knice subgroups of $G$.

ProOF. By Theorem 4.8, $H$ itself is a $k$-group. But as noted earlier, it is a consequence of Theorem 3.7 that summands of $k$-groups are knice subgroups. The conclusion that $A$ and $B$ are knice in $G$ follows from $4.10(\mathrm{i})$.

5. The third axiom of countability. A torsion free group $G$ is said to satisfy the third axiom of countability with respect to knice subgroups provided there is a family $C$ of knice subgroups of $G$ such that the following three conditions hold:

(0) $0 \in C$.

(1) $C$ is closed with respect to the group union of an arbitrary number of groups.

(2) If $A \in C$ and $S$ is a countable subset of $G$, then there exists a $B \in C$ such that $B / A$ is countable and $B \supseteq\langle A, S\rangle$.

PROPOSITION 5.1. If $C$ is a family of knice subgroups of $G$ satisfying (0), (1) and (2), then $C$ contains a subfamily $C^{\prime}$ of pure knice subgroups satisfying (0), (1) and (2).

ProOF. By Theorem 2 of [5], there is a family $P$ of pure subgroups of $G$ satisfying (0), (1) and (2). Let $C^{\prime}=C \cap P$. Clearly $C^{\prime}$ satisfies (0) and (1). Now suppose $A \in C^{\prime}$ and let $S$ be a countable subset of $G$. Exploiting condition (2) for $C$ and $P$ separately, we generate inductively a nested ascending sequence

$$
B_{0} \subseteq C_{0} \subseteq B_{1} \subseteq C_{1} \subseteq \cdots \subseteq B_{n} \subseteq C_{n} \subseteq \cdots,
$$

where $\langle A, S\rangle \subseteq B_{0}, B_{0} / A$ is countable, each $C_{n}$ is in $P$ and both $C_{n} / B_{n}$ and $B_{n+1} / C_{n}$ are countable for all $n$. Then $B=\bigcup_{n<\omega} B_{n}=\bigcup_{n<\omega} C_{n}$ is in $C^{\prime},\langle A, S\rangle \subseteq$ $B$ and $B / A$ is countable. Thus $C^{\prime}$ satisfies (2), as desired.

THEOREM 5.2. If $G$ is a torsion free group satisfying the third axiom of countability with respect to knice subgroups, then any direct summand of $G$ also satisfies the third axiom of countability with respect to knice subgroups.

Proof. Suppose $G=H \oplus K$ and let $C$ be a family of knice subgroups of $G$ satisfying (0), (1) and (2). By 5.1, we may assume that the members of $C$ are 
pure and knice in $G$. Let $C^{\prime}$ consist of all those subgroups $A$ of $H$ for which there corresponds an $N \in C$ such that $N=A \oplus(N \cap K)$. It is a familiar fact (see, e.g., the proof of 81.5 in [3]) that $C^{\prime}$ also satisfies (0), (1) and (2). It remains to show that the members of $C^{\prime}$ are knice in $H$. But this is an easy consequence of 4.3 and 3.7. Indeed suppose $A \in C^{\prime}$ and $N=A \oplus(N \cap K) \in C$. It is then a routine argument to show that $N$ being balanced in $G$ implies that $A$ is balanced in $H$; while $H / A$ is a $k$-group since it is canonically isomorphic to a direct summand of the $k$-group $G / N$.

Given the well-known third axiom of countability characterization of simply presented torsion groups, it should not be surprising that the class of torsion free groups we have introduced in this section consists precisely of the simply presented ones. This is indeed the content of our next theorem.

THEOREM 5.3. A torsion free group satisfies the third axiom of countability with respect to knice subgroups if and only if it is completely decomposable.

ProOF. Assume first that $G=\bigoplus_{i \in I} G_{i}$, where each $G_{i}$ is a torsion free rank one group. For each subset $J$ of $I$, let $G(J)=\sum_{i \in J} G_{i}$. As a summand of the $k$-group $G$, each $G(J)$ is a knice subgroup of $G$. If $C^{\prime}$ consists of all $G(J)$ as $J$ ranges over all subsets of $I$, then it is clear that $C$ satisfies (0), (1) and (2).

Conversely, assume that $C$ is a family of pure knice subgroups of $G$ that satisfies $(0),(1)$ and (2). It is routine to show that $G$ is the union of a well-ordered family $\left\{H_{\alpha}\right\}_{\alpha<\mu}$ satisfying the following conditions:

(i) $H_{0}=0, H_{\alpha} \subseteq H_{\beta}$ if $\alpha<\beta$ and $H_{\alpha}=\bigcup_{\beta<\alpha} H_{\beta}$ whenever $\alpha$ is a limit ordinal;

(ii) For all $\alpha<\mu, H_{\alpha} \in C$ and $H_{\alpha+1} / H_{\alpha}$ is countable.

The proof is completed by showing that, for each $\alpha, H_{\alpha+1}=H_{\alpha} \oplus L_{\alpha}$, where $L_{\alpha}$ is completely decomposable; for if this can be established, it will follow that $G=\bigoplus_{\alpha<\mu} L_{\alpha}$. Thus, by $3.5,4.3$ and 4.4, it is enough to prove that $H_{\alpha}$ is knice in $H_{\alpha+1}$ for each $\alpha$. But since $H_{\alpha}$ is balanced in $G$ and $H_{\alpha+1} / H_{\alpha}$ is pure in $G / H_{\alpha}$, it is routine to verify that $H_{\alpha}$ is balanced in $H_{\alpha+1}$. By 4.3 it remains only to prove that $H_{\alpha+1} / H_{\alpha}$ is a $k$-group. It is, however, a triviality to show that $H_{\alpha+1}$ being a pure knice subgroup $G$ implies that $H_{\alpha+1} / H_{\alpha}$ is a pure knice subgroup of the $k$-group $G / H_{\alpha}$. Finally, an application of 4.8 completes the proof.

Notice that 5.2 and 5.3 combine to yield the Baer-Kulikov-Kaplansky theorem which asserts that summands of completely decomposable groups are themselves completely decomposable. Of course, theorems insuring that certain subgroups of completely decomposable groups are completely decomposable are difficult to come by, and all too often such theorems involve artificial assumptions concerning the set of types assumed by elements of the containing group. As a final application of the ideas developed in this paper, however, we shall present a fairly general criterion concerning subgroups of completely decomposable groups which can be paraphrased as follows: Separable groups of cardinality at most $\aleph_{1}$ have balanced projective dimension $\leq 1$.

THEOREM 5.4. If $H$ is a balanced subgroup of the completely decomposable group $G$ and if $G / H$ is a separable group of cardinality not exceeding $\aleph_{1}$, then $H$ is completely decomposable. 
PROOF. We begin our proof with a few preliminary observations. First we note that a separable group $K$ of cardinality $\aleph_{1}$ is the union of a smooth wellordered chain $\left\{K_{\alpha}\right\}_{\alpha<\omega_{1}}$, where each $K_{\alpha}$ is a countable, separable pure subgroup of $K$. Indeed beginning with a well-ordering $\left\{x_{\alpha}\right\}_{\alpha<\omega_{1}}$ of the elements of $K$, we construct the $K_{\alpha}$ 's inductively in such a fashion that (a) $x_{\alpha} \in K_{\alpha+1}$ and (b) each finite subset $S$ of $K_{\alpha}$ can be imbedded in a finite rank summand $A$ of $K$ with $A \subseteq K_{\alpha}$. Assume that the requisite $K_{\alpha}$ 's have been constructed for all $\alpha<\beta$. If $\bar{\beta}$ is a limit ordinal, we let $K_{\beta}=\bigcup_{\alpha<\beta} K_{\alpha}$ and observe that condition (b) is inherited by $K_{\beta}$. One the other hand, if $\beta=\alpha+1$ for some $\alpha$, then we choose an enumeration $\left\{y_{n}\right\}_{n<\omega}$ of the elements of $K_{\alpha}$ and take $K_{\beta}=\bigcup_{n<\omega} A_{n}$, where the $A_{n}$ 's form an ascending sequence of finite rank summands of $K$ with $A_{n}$ containing $\left\{y_{0}, \ldots, y_{n}, x_{\alpha}\right\}$.

Our remaining preliminary observations involve the notion of global compatibility in the sense of [6]. If $A$ and $B$ are subgroups of $G$, then we write $A \| B$ to indicate that the following condition is satisfied: If $(a, b) \in A \times B$ and if $s \leq|a+b|$ for some height sequence $s$, then there exists a $b^{\prime} \in A \cap B$ such that $s \leq\left|a+b^{\prime}\right|$. If $H$ is a balanced subgroup of $G$ and if $S$ is a countable subset of $G$, then there is a countable subgroup $B$ of $G$ such that $S \subseteq B$ and $B \| H$ (see the proof of Lemma 1 in [6]). Finally, we wish to note that $A \cap H$ will be a balanced subgroup of $A$ provided $H$ is balanced in $G$ and $A$ is a pure subgroup of $G$ with $A \| H$. Indeed suppose $s=|a+A \cap H|$, where heights are computed in $A / A \cap H$, or equivalently in $G / A \cap H$ since $A$ is pure. But then $|a+H| \geq s$ and since $H$ is balanced in $G$, there is an $h \in H$ such that $|a+h|=|a+H|$. Recalling that $A \| H$, we see that $\left|a+h^{\prime}\right| \geq s$ for some $h^{\prime} \in A \cap H$ and it follows that $A \cap H$ is balanced in $A$.

We shall first prove the theorem under the further restriction that $|G| \leq \aleph_{1}$. Then as the proof of 5.3 clearly indicates, it suffices to show that $H$ is the union of a family $C$ of countable pure knice subgroups where $C$ satisfies $(0),(2)$ and the following weakened version of (1):

$\left(1^{\prime}\right) C$ is closed under the union of countable chains.

We fix a direct decomposition $G=\bigoplus_{i \in I} G_{i}$, where each $G_{i}$ is a rank one group, and we let $G(J)=\sum_{i \in J} G_{i}$ whenever $J$ is a subset of $I$. Since $G / H$ is separable, one of our preliminary observations tells us that $G / H$ is the union of a family $D$ of countable, separable pure subgroups where $D$ also satisfies the closure property $\left(1^{\prime}\right)$. We shall call a subset $J$ of $K$ "special" provided $J$ satisfies the following conditions:

(i) $J$ is countable;

(ii) $G(J) \| H$;

(iii) $G(J)+H / H$ is in $D$.

Given the inductive nature of (ii) and our earlier observations, a standard backand-forth argument shows that $\{G(J): J$ is "special" $\}$ satisfies (0), (1') and (2). Thus if we set $H(J)=H \cap G(J)$, the family $C=\{H(J): J$ is "special" $\}$ inherits the properties (0), $\left(1^{\prime}\right),(2)$. It remains to show that the members of $C$ are knice in $H$. Initially we recognize each $H(J)$ with $J$ "special" as a knice subgroup of $G(J)$ by 4.3 since, as observed in the preceding paragraph, (ii) implies that $H(J)$ is balanced in $G(J)$ and (iii) implies that $G(J) / H(J) \simeq G(J)+H / H$ is a $k$-group. Since the summands $G(J)$ of the $k$-group $G$ are knice, $4.10(\mathrm{i})$ tells us that the members of 
C are knice in $G$. Finally, note that 4.10(iv) then implies that the $H(J)$ 's with $J$ "special" are actually knice in $H$.

We still have the problem of removing the cardinality restriction on $G$. Fortunately, this difficulty can be overcome by a routine variant of Schanuel's trick. First note that, since $|G / H| \leq \aleph_{1}$ and $G$ is completely decomposable, $G / H$ is canonically the image of a summand $G_{0}$ of $G$ with $\left|G_{0}\right| \leq \aleph_{1}$. Furthermore, we can choose $G_{0}$ such that each element of $G / H$ has a preimage in $G_{0}$ with the same height sequence and therefore $H_{0}=H \cap G_{0}$ will be balanced in $G_{0}$. By the case covered by our restricted version of the theorem, $H_{0}$ is completely decomposable. Since it is routine that pullbacks of balanced exact sequences are balanced exact, we obtain two balanced exact sequences $0 \rightarrow H \rightarrow K \rightarrow G_{0} \rightarrow 0$ and $0 \rightarrow H_{0} \rightarrow K \rightarrow G \rightarrow 0$, where $K$ is the obvious subgroup of $G \oplus G_{0}$. Since $G$ and $G_{0}$ are completely decomposable, these sequences split and we have an isomorphism $H \oplus G_{0} \simeq H_{0} \oplus G$. The Baer-Kulikov-Kaplansky theorem yields the desired conclusion that $H$ is completely decomposable.

COROLLARY 5.5. If $G$ is a completely decomposable group of cardinality not exceeding $\aleph_{1}$, then every strongly balanced subgroup of $G$ is completely decomposable.

\section{REFERENCES}

1. R. Baer, Abelian groups without elements of finite order, Duke Math. J. 3 (1937), 68-122.

2. L. Fuchs, Summands of separable abelian groups, Bull. London Math. Soc. 2 (1970), 205208.

3. _ Infinite abelian groups, Vol. II, Academic Press, New York, 1973.

4. P. Griffith, Infinite abelian groups, Univ. of Chicago Press, Chicago, 1970.

5. P. Hill, The third axiom of countability for abelian groups, Proc. Amer. Math. Soc. 82 (1981), 347-350.

6. , Isotype subgroups of totally projective groups, Lecture Notes in Math., vol. 874, Springer-Verlag, New York, 1981, pp. 305-321.

7. P. Hill and C. Megibben, On the theory and classification of abelian p-groups, Math. Z. 190 (1985), 17-38

8. __ Axiom 3 modules, Trans. Amer. Math. Soc. 295 (1986), 715-734.

9. K. Rangaswamy, On strongly balanced subgroups of separable torsion-free abelian groups, Lecture Notes in Math., vol. 1006, Springer-Verlag, New York, 1983, pp. 268-274.

Department of Mathematics, Auburn University, Auburn, Alabama 36849

Department of MATHEMATICS, VANDERBILT UNIVERSity, NASHVIlle, TENNESSEe 37235 (Current address of Charles Megibben)

Current address (Paul Hill): Department of Mathematics, Baylor University, Waco, Texas 76798 